\title{
Acarbose for type 2 diabetes prevention
}

André J Scheen

Division of Diabetes, Nutrition and Metabolic Disorders, Department of Medicine, CHU Sart Tilman, B-4000 Liège, Belgium

Sir-Jean-Louis Chiasson and colleagues (June 15, p 2072) ${ }^{1}$ report that after a mean follow-up of 3.3 years, patients with impaired glucose tolerance (IGT) receiving acarbose, an $\alpha$-glucosidase inhibitor, less frequently developed diabetes and more frequently showed a reversion to normal glucose tolerance than did patients assigned placebo. These conclusions could be used to delay development of type 2 diabetes in patients with IGT. One key question is, however, whether these positive results could be interpreted as a real prevention of the disease or only as a delay in its progression or simply a masking effect due to an acute metabolic effect of the drug.

The curves of the cumulative probability of remaining free of diabetes over time are almost parallel beyond year 1 in the acarbose and placebo groups, which may suggest that acarbose retards rather than really prevents type 2 diabetes. In the Diabetes Prevention Program (DPP), ${ }^{2}$ after an average follow-up of $2 \cdot 8$ years, treatment with metformin reduced the rate of diabetes compared with placebo in people with IGT. Thus, the results of both pharmacological interventions were similar, although less impressive that those of lifestyle intervention.

In the DPP, however, the final oral glucose tolerance test was done while the patients still were taking metformin with no wash-out period. Short-term administration of metformin for only $48 \mathrm{~h}$ is sufficient to improve glucose tolerance in patients with IGT; ${ }^{3}$ therefore, a treatment effect rather than a true prevention of type 2 diabetes could not be excluded in the DPP.

Acarbose also exerts acute effects on glucose tolerance in people with and without diabetes. ${ }^{4}$ However, at the end of Chiasson and colleagues' trial, and by contrast with DPP, all patients were given single-blind placebo for 3 months, after which all outcome measures were repeated. During this period, the rate of diabetes in patients who had not converted before was higher in the group originally assigned acarbose than that first randomised to placebo, so that the final difference in conversion to diabetes was reduced by more than a third compared with that noted under active treatment 3 months before. This observation suggests that a treatment effect of acarbose is present (as most probably is with metformin in the DPP), which could simply mask diabetes and disappear as soon as drug administration is stopped.

By contrast, preservation of B-cell function and protection from type 2 diabetes persisted 8 months after interruption of troglitazone in the recent TRIPOD trial, ${ }^{5}$ a finding that suggests a long-lasting protection effect with thiazolidinediones. The question remains open whether the results of the STOP-NIDDM trial with acarbose, as for those of the DPP with metformin, correspond to preventing, delaying, or partly masking effects of type 2 diabetes.

\section{Bibliography}

1 Chiasson J-L, Josse RG, Gomis R, Hanefeld M, Karasik A, Laakso M, for the STOP-NIDDM Trial Research Group. Acarbose for prevention of type 2 diabetes mellitus: the STOP-NIDDM randomised trial. Lancet 2002; 359: 2072-77.

2 Diabetes Prevention Program Research Group. Reduction in the incidence of type 2 diabetes with lifestyle intervention or metformin. $N$ Engl J Med 2002; 346: 393-403.

3 Scheen AJ, Letiexhe MR, Lefèbvre PJ. Short administration of metformin improves insulin sensitivity in obese android subjects with impaired glucose tolerance. Diabet Med 1995; 12: 985-89.

4 Scheen AJ. Clinical efficacy of acarbose in diabetes mellitus: a critical review of controlled trials. Diabetes Metab 1998; 24: 311 -20.

5 Buchanan TA, Xiang AH, Peters RK, et al. Protection from type 2 diabetes persists in the TRIPOD cohort eight months after stopping troglitazone. Diabetes 2001; 50 (suppl 2): A81 (abstr). 